\title{
Giant Colonic Diverticulum of the Sigmoid with an Unusual Localization
}

\author{
Misha R.M. Frenken ${ }^{a} \quad$ Lisanne M. Koenjer $^{b} \quad$ Marieke G.J. Versteegen $^{b}$ \\ Jeroen F. Vogelaar ${ }^{a}$ \\ aDepartment of General Surgery, VieCuri Medical Center, Venlo, The Netherlands; \\ ${ }^{b}$ Department of Emergency medicine, VieCuri Medical Center, Venlo, The Netherlands
}

\section{Keywords}

Giant colonic diverticula · Diverticular disease · Diverticulosis · Sigmoid

\begin{abstract}
Diverticular disease is a common disorder of the colon. A rare complication of diverticulosis is a giant colonic diverticulum (GCD) defined as a diverticulum measuring $>4 \mathrm{~cm}$. It most often affects the sigmoid colon. We report a case of a 68-year-old male presenting at the emergency department with a giant diverticulum of the sigmoid with an unusual size and migration to the right hemi-abdomen. Segmental colectomy with en-bloc diverticular resection and primary anastomosis seems to be the best treatment according to the available current literature.
\end{abstract}

\section{Introduction}

Colonic diverticula can cause symptoms in case of inflammation, bleeding or when the diverticulum evolves into a giant colonic diverticulum (GCD). A GCD is defined as a diverticulum larger than $4 \mathrm{~cm}$ in size, arising from a valve function in which gas enters the diverticulum but is unable to leave [1].

Most patients present with 1 single (90\% of the cases) giant diverticulum, with the sigmoid as the most common location (80-90\%) [1]. The mean age of people with a giant colonic diverticulum is between the 60 and 79 years, with an average size of 4-9 cm [1]. Patients present with complaints as abdominal pain, constipation and vomiting. A tender or non-tender abdominal mass can be found during physical examination. Some patients present 
Fig. 1. Computed tomography; coronal view of the giant cell diverticulum located in the right upper quadrant due to herniation of the sigmoid through the right mesocolon. Hemangioma of the liver. Aortic calcifications.

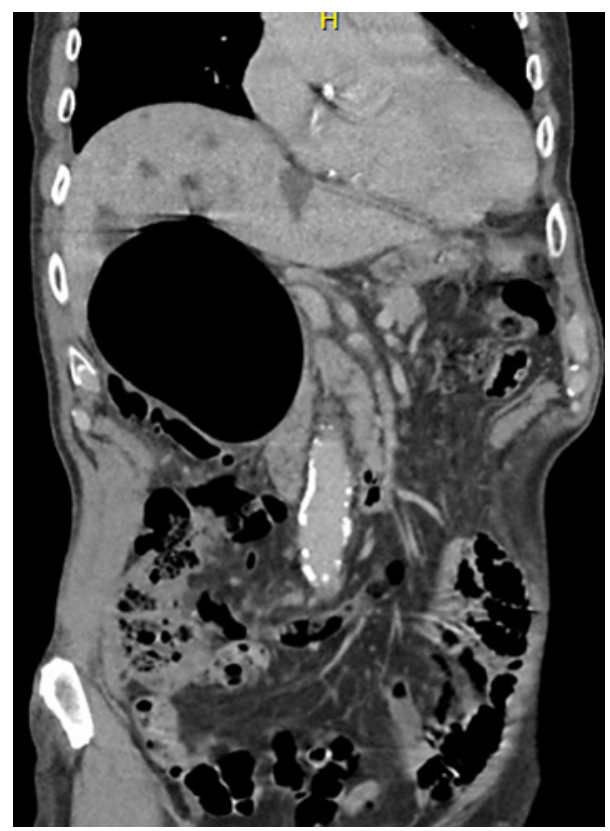

after complications of the GCD such as perforation and/or abscess formation [1, 2]. In this report, we describe a GCD of the sigmoid with migration to the right upper abdomen; we did not find other cases in the literature which described this form of migration.

\section{Case Presentation}

A 68-year-old male patient presented at the emergency department with worsening right upper quadrant abdominal pain, which started 1 week ago. Besides moderate mental impairment, the patients' medical history only mentioned a cerebral infarction 3 years ago, with a slight residual loss of strength in his right arm.

The patient complained of right upper abdominal pain, without nausea or vomiting. His appetite was decreased. No changes in micturition or defecation pattern were observed.

Physical examination revealed normal vital parameters. During abdominal exam, there was a mass visible in his right hemi-abdomen, which was painful and non-tender during palpation. Besides a slightly elevated C-reactive protein of $43 \mathrm{mg} / \mathrm{L}$, blood count showed no abnormalities. On the left side, there was a possible encapsulated rupture or dissection.

An additional CT scan with contrast was made (See Fig. 1, 2), which showed no aortic dissection as suggested on the ultrasound, but an infrarenal ectasia of $3.3 \mathrm{~cm}$ with thrombus formation of the aortic wall. An unusual route of the sigmoid was seen with herniation through the right mesocolon, where it crossed the right psoas ending paracolic to the right in an air-filled dilated structure with a size of about $12 \mathrm{~cm}$ in diameter. The precise connection between the sigmoid and the air-filled structure in the right upper abdomen was not clearly visible. There was no dilatation of the remaining colon, no pneumatosis, nor signs of ischemia.

The patient underwent surgery because of the suspected internal herniation on the same day of admission. A median laparotomy was carried out and revealed a giant diverticulum of the sigmoid of $14 \mathrm{~cm}$ in diameter (See Fig. 3), which was migrated with the sigmoid and was

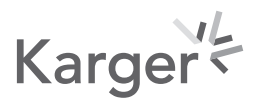


Fig. 2. Computed tomography; transversal view.

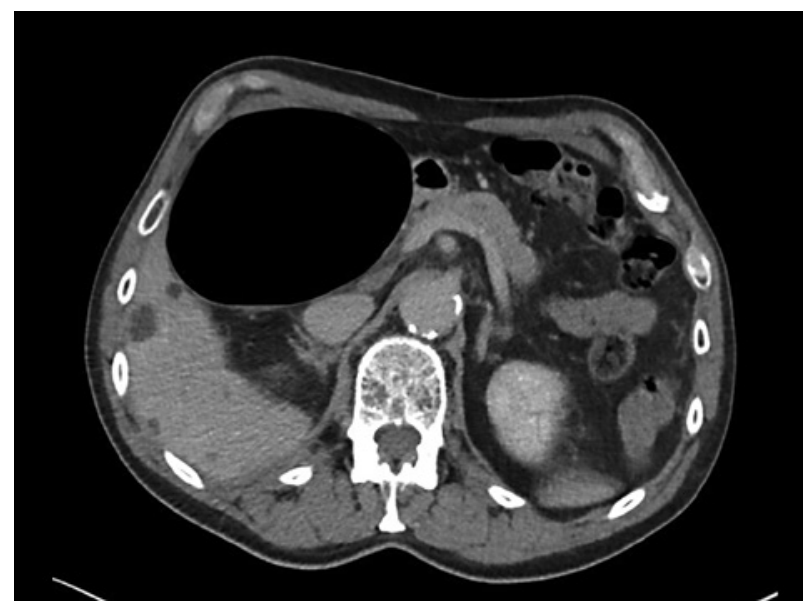

Fig. 3. Perioperative image; a median laparotomy is performed and revealed a giant airfilled diverticulum of the sigmoid of $14 \mathrm{~cm}$ in diameter.

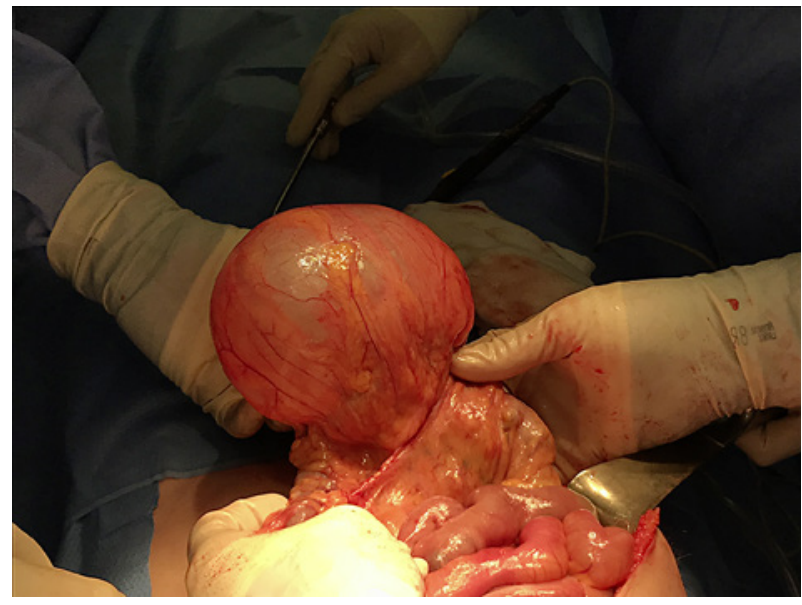

located just beneath the liver. There was no herniation, strangulation, or ischemia of the colon. A small sigmoid resection $(9 \mathrm{~cm}$ ), including the $14-\mathrm{cm}$ large giant diverticulum (en-bloc) with a side-to-side stapled anastomosis was performed.

Macroscopic and histologic examination revealed diverticulosis and focal active diverticulitis with perforation in a solitary peritoneal pseudocyst. In addition, there was a tubular adenoma $(0.7 \mathrm{~cm})$ with low-grade dysplasia. The inner surface only consisted of chronic inflammatory tissue without an epithelial layer.

\section{Discussion}

This case describes migration of a giant cell diverticulum of the sigmoid through the mesocolon into the right upper abdomen. This proves that if the pressure inside a sigmoid diverticulum gets high enough by accumulation of air through valve function, the diverticulum can migrate causing nonspecific complaints in other parts of the abdomen. We did not find other cases in the literature which described this form of migration to the right upper quadrant. 
Histologically, there are 3 different types of GCD described; a pseudo diverticulum, an inflammatory giant diverticulum and a true diverticulum [3]. In case of a pseudo diverticulum, the diverticulum gradually increases in size. Histologically remnants of the muscularis mucosa and muscularis propria may be found. Most of the time, the mucosa is not completely intact. The second type of GCD is the inflammatory giant diverticulum (in case of our report). This diverticulum results from a local perforation of the mucosa with an abscess cavity, which maintains a connection with the lumen of the colon. Due to this perforation, a valve function can occur and leads to air pressure build up inside the diverticulum as described in our case. In this type, there are no mucosal remnants found histologically, since it only consists of reactive scar tissue.

The third type is a true diverticulum, in which the wall of the diverticulum consists of the same layers as the intestinal wall (serosa, muscularis, submucosa, and mucosa). The layers are in continuity with the gut lumen. In $2 \%$ of the cases described in the literature, a carcinoma is found inside the GCD [1].

The preferred treatment of GCD is a segmental colectomy with en-bloc diverticular resection and primary anastomosis [2], with or without temporary colonic anastomosis. A Hartmann's resection could be performed in high-risk patients, severe diverticulitis of the sigmoid or in emergency settings.

Nonsurgical treatment in GCD is not recommended because of the substantial risk of complications and risk of carcinoma development $[1,2]$.

\section{Conclusion}

GCD is a rare complication of diverticulosis and is sometimes hard to diagnose, in case of our patient. An inflammatory giant cell diverticulum is able to migrate through the abdomen by air pressure build up causing non-specific abdominal pain. Segmental colectomy with en-bloc diverticular resection and primary anastomosis seems to be the best treatment according to the available current literature.

\section{Statements of Ethics}

Written informed consent was obtained from the patient for publication of this case report and any accompanying images; therefore, the paper is exempt from ethical committee approval.

\section{Conflict of Interest Statement}

The authors have no conflicts of interest to declare.

\section{Funding Sources}

The author(s) received no specific funding for this work. 


\section{Author Contributions}

All listed authors contributed substantially to the work, and that the work is an original contribution. Misha R.M. Frenken contributed to writing - review and editing. Lisanne M. Koenjer contributed to writing - original draft preparation and project administration. Marieke G.J. Versteegen involved in supervision. Jeroen F. Vogelaar contributed to conceptualization and supervision.

\section{References}

1 Steenvoorde P, Vogelaar FJ, Oskam J, Tollenaar RA. Giant colonic diverticula. Review of diagnostic and therapeutic options. Dig Surg. 2004;21(1):1-6; discussion 6. Epub 2003 Nov 19. PMID: 14631129.

2 Nigri G, Petrucciani N, Giannini G, Aurello P, Magistri P, Gasparrini M, et al. Giant colonic diverticulum: clinical presentation, diagnosis and treatment: systematic review of 166 cases. World J Gastroenterol. 2015;21(1): 360-8.

3 McNutt R, Schmitt D, Schulte W. Giant colonic diverticula - three distinct entities. Report of a case. Dis Colon Rectum. 1988 Aug;31(8):624-8. 